\title{
Human Papillomaviruses and cervico vaginal co-infections in a population of Molise
}

\author{
Massimiliano Scutellà,Valentina Felice, Giovanni Cuzzone, Pasqualina Picciano, Rita Gisi, \\ Amelia Di Stefano \\ Laboratorio Analisi P.O. “A. Cardarelli” Campobasso, Azienda Sanitaria Regione Molise
}

Key words: HPV infection, Bacterial vaginosis, Ureaplasma urealyticum

\section{Papilloma Virus e co-infezioni cervico vaginali in una popolazione del Molise}

\section{SUMMARY}

High grade HPV infections and persistence are the strongest risk factors for cervical cancer. Nevertheless other genital microrganism may be involved in the progression of HPV associated lesions. Bacterial vaginosis (BV), an alteration of vaginal flora involving a decrease in Lattobacilli and predominance of anaerobic bacteria, is the most common cause of vaginal complaints for women of childbearing age.

Although the specific role of anaerobic bacteria in the pathogenesis of cervical cancer has not been fully elucidated, it has been suggested that HPV infection alone may not be sufficient for full induction of cervical carcinogenesis, and that the simultaneous presence of cervico vaginal bacteria may increase the risk of neoplastic progression.

In this cross-sectional study on 356 enrolled outpatients, statistical analyses revealed a significant association of HPV with Ureaplasma urealyticum detection. Although BV was mildly associated with HPV $(O R=2.4)$, it was more common among the HPV positive women. These data confirm that screening for genital infections may be important to reveal the simultaneous presence of different sexually transmitted microrganisms. These results suggest and emphasize the value of the screening for genital infections in HPV positive patients in order to decrease the presence of the other microrganisms and to reduce the probable synergistic effects of coinfections. Prevention is important not only to avoid other sexually transmitted disease and their sequelae, but also to reduce the influence of concomitant microrganisms on HPV infection.

\section{INTRODUZIONE}

L'infezione da Papilloma Virus (HPV) svolge un ruolo primario nella carcinogenesi della cervice uterina $(7,10,11)$.

Infatti, indagini epidemiologiche e molecolari hanno dimostrato inequivocabilmente che l'infezione da HPV sostenuta da ceppi ad alto rischio oncogeno è il principale fattore causale nell'avvio della trasformazione progressiva che porta alla neoplasia cervicale intraepiteliale e al cancro del collo dell'utero $(16,17)$. La prevalenza di HPV a livello mondiale è pari al $27.4 \%$, e la frequenza di donne con ceppi ad alto rischio oncogeno è più alta rispetto a quella con ceppo a basso rischio (rispettivamente $18.7 \%$ e $7.5 \%$ ) (14).

E generalmente riconosciuto che cofattori diversi sono coinvolti nello sviluppo della displasia nelle donne infette da HPV. Fattori come il genotipo specifico, la carica virale e la coinfezione con più ceppi HPV, sono coinvolti nella progressione di lesioni cervicali precancerose $(5,8)$. Inoltre $i$ fattori ambientali tra cui il fumo, i contraccettivi orali a lungo termine, l'immunosoppressione, il deficit di vitamine, ma soprattutto la coinfezione con altri agenti di cervico-vaginiti sono considerati come potenzialmente coinvolti nella genesi della malattia $(20,21)$. Diversi microrganismi cervico-vaginali hanno mostrato, in associazione con l'infezione da HPV, un effetto sinergico nella formazione di lesioni cervicali displastiche $(6,13$, $15,22)$.

Infatti, molti agenti patogeni sono stati studiati sia come fattore di rischio che come cofattori di HPV nello sviluppo delle lesioni precancerose e cancerose della cervice uterina, con la conclusione che alcuni agenti sessualmente trasmessi potrebbero essere responsabili dello sviluppo di lesioni cervicali sia direttamente, sia mediante la produzione di metaboliti o altre sostanze carcinogenetiche, che aumentano la suscettibilità dell'epitelio infiammatorio (1).

L'infezione da HPV può essere influenzata da una comune infezione vaginale curabile che altera l'ecosistema vaginale e $\mathrm{i}$ suoi innati meccanismi di protezione nei confronti di infezioni e malattie (10). La vaginosi batterica (BV) è un'infezione del basso tratto genitale femminile e rappresenta l'infezione vaginale tra le più diffuse nelle donne 
in età riproduttiva. Si tratta di una condizione caratterizzata dall'alterazione del normale ecosistema vaginale e del suo $\mathrm{pH}(2,19)$. In particolare una diminuzione della flora lattobacillare determina una variazione del $\mathrm{pH}$ e la proliferazione di batteri opportunisti come Gardnerella vaginalis, Mobiluncus spp, Bacteroides spp, Prevotella spp, Peptostreptococcus spp, Mycoplasma hominis e Ureaplasma urealyticum che causano un aumento del $\mathrm{pH}$ vaginale $(2,18)$.

Le proteasi batteriche degradano il muco secreto dalla cervice facilitando la penetrazione dei patogeni nella barriera epiteliale protettiva (3).

Anche se il ruolo specifico di questi microrganismi nella patogenesi del cancro del collo dell'utero non è stato completamente chiarito, è stato proposto che la sola infezione da HPV potrebbe non essere sufficiente per una completa induzione della carcinogenesi cervicale, e che la simultanea presenza di microrganismi causa di cervico-vaginiti potrebbe aumentare il rischio di progressione neoplastica.

Lo scopo di questo studio retrospettivo è stato quello di valutare la prevalenza di coinfezioni da HPV in una popolazione molisana.

\section{MATERIALI E METODI}

\section{Studio sulla popolazione molisana}

Lo studio si riferisce alla raccolta di dati anamnestici, desunti attraverso la compilazione di una dettagliata scheda riportante dati clinici, e laboratoristici retrospettivi analizzati in tre anni di attività routinaria da maggio 2009 a dicembre 2011. Le donne inserite nello studio afferivano al Servizio di Ginecologia del P.O. "A. Cardarelli" di Campobasso per sottoporsi ai test routinari di screening e per la ricerca di HPV-DNA.

I campioni cervicali per la rilevazione di HPVDNA sono stati prelevati mediante scraping cervicale, mentre i campioni per le indagini batteriologiche mediante tamponi cervico vaginali.

L'esame citologico è stato eseguito contestualmente o nei mesi precedenti e classificato secondo il sistema Bethesda, che prevede la classificazione dei seguenti stati: negativo, cellule squamose atipiche di significato indeterminato (ASCUS), cellule ghiandolari atipiche di significato indeterminato (AGUS), lesioni intraepiteliali di basso (LSIL) o alto grado (H-SIL).

Le donne inserite nello studio non erano in stato di gravidanza, avevano un utero intatto e nessun riferimento corrente per l'isterectomia, non riportavano l'uso di farmaci vaginali nei precedenti tre giorni e rispondevano ad un questionario strutturato su caratteristiche socio-demografiche, sul comportamento sessuale, e sulla storia riproduttiva.

\section{Identificazione batterica}

Nella diagnostica delle Infezioni Sessualmente Trasmissibili (IST) sono state identificate le seguenti specie microbiche:

\section{- Vaginosi Batterica}

Presenza di clue-cells all'esame microscopico con colorazione di Gram che evidenzia flora batterica cocco-bacillare Gram variabile sulla superficie di cellule epiteliali vaginali.

L'osservazione microscopica viene valutata mediante lo score di Nugent (valutazione semiquantitativa) che permette una diagnosi dell'infezione distinguendo una franca vaginosi batterica (BV) (score da 7 a 10) da quelle forme border line di BV (score da 3 a 6 ).

\section{- Miceti}

Crescita su Sabouraud Agar e speciazione mediante l'utilizzo di prove biochimiche.

\section{- Streptococcus agalactiae (SGB)}

Isolato su Agar-Sangue. Identificazione di colonie con tipica beta-emolisi, anche se alcuni stipiti eccezionalmente sono non emolitici.

L'identificazione previa estrazione e rivelazione mediante agglutinazione al lattice degli antigeni polisaccaridici gruppo-specifici che può essere preceduta da quella presuntiva basata sulla positività al test dell'idrolisi dell'ippurato.

\section{- Trichomonas vaginalis}

Esame microscopico a fresco, coltura in terreno selettivo in fase liquida e successiva osservazione microscopica a fresco.

\section{- Neisseria gonorrhoeae}

Crescita su terreni di coltura Agar Cioccolato e Agar Thayer-Martin modificato preriscaldati in termostato per $5^{\prime}$ e incubati a $37^{\circ} \mathrm{C}$ in atmosfera di $\mathrm{CO}_{2}$ al $5 \%$ per $48 \mathrm{~h}$. Esame microscopico previa colorazione di Gram che evidenzia diplococchi Gram negativi a chicco di caffé e successiva identificazione mediante prove biochimiche.

\section{- Chlamydia trachomatis}

Metodica molecolare che prevede l'estrazione del DNA batterico e la successiva amplificazione e rivelazione mediante PCR real-time.

\section{- Mycoplasma/Ureaplasma}

Metodica colturale colorimetrica che prevede l'utilizzo di un terreno di trasporto/coltura e una galleria contenente substrati di identificazione e antibiotici liofilizzati che devono essere reidratati con il brodo.

La presenza di substrati specifici (urea per Ureaplasma spp e arginina per Mycoplasma hominis) e di un indicatore (rosso-fenolo) permette, in caso di coltura positiva, di visualizzare un cambiamento di colore del brodo conseguente all'aumento del $\mathrm{pH}$. Inoltre, la stessa variazione colorimetrica permette di determinarne la carica ed il comportamento dello stipite isolato in rela- 
zione agli antibiotici di uso comune.

Il metodo permette la valutazione semiquantitativa del cut-off di carica (10.000 UFC/mL) sopra il quale si può attribuire un ruolo patogeno al microrganismo. I valori al di sotto sono considerati come indice di semplice commensalismo.

\section{Identificazione e tipizzazione di virus HPV}

I campioni raccolti mediante scraping cervicali e conservati in tampone fosfato salino (PBS), sono stati centrifugati a bassa velocità, e il pellet delle cellule sottoposto a processo di estrazione del DNA utilizzando il kit QIAamp Mini Elute Virus Spin Kit (Qiagen, Milano Italia).

Per l'identificazione del virus HPV sono state utilizzate metodiche molecolari che prevedono l'amplificazione del DNA mediante PCR Real Time.

La tipizzazione dei ceppi è stata eseguita con metodica sempre molecolare che prevede l'amplificazione mediante PCR e successiva ibridazione molecolare su striscia (Line Probe Assay).

\section{Metodi Statistici}

Allo scopo di dimostrare l'associazione tra la coinfezione e la positività all'HPV è stato utilizzato, in questo studio retrospettivo, un indice statistico: il calcolo dell'odds ratio (OR).

Prima di dichiarare l'esistenza di un rapporto causa-effetto tra HPV e coinfezione e per escludere che la differenza sia dovuta al caso, è stato eseguito un test di significatività statistica: il test del Chi-quadro per il quale la differenza risulta significativa se $p<0.025$.

Un altro importante indice di associazione utilizzato in questo studio è il rischio attribuibile (RA). È stato inoltre calcolato il rischio assoluto di sviluppare una data malattia per un individuo esposto ad un fattore di rischio.

\section{RISULTATI}

Da maggio 2009 a dicembre 2011 sono afferite all'ambulatorio di Ginecologia del P.O. "Cardarelli di Campobasso", 356 donne sottoposte a tampone vaginale/endocervicale e a ricerca di HPV-DNA.

L'età delle donne era compresa tra 16 e 70 anni, di cui il $12 \%$ di età inferiore ai 25 anni, il $26 \%$ tra 25 e 30 anni, il $21 \%$ tra 31 e 35 e infine il $41 \%$ di età superiore ai 35 anni.

Le pazienti sono state divise in due gruppi sulla base della positività del test molecolare per HPVDNA: 99 sono risultate positive al test (28\%) e 257 negative $(72 \%)$.

La figura I mostra l'andamento della prevalenza dell'infezione divisa per fascia di età in cui si evidenzia un picco d'infezione nelle donne di età compresa tra 25 e 30 anni.

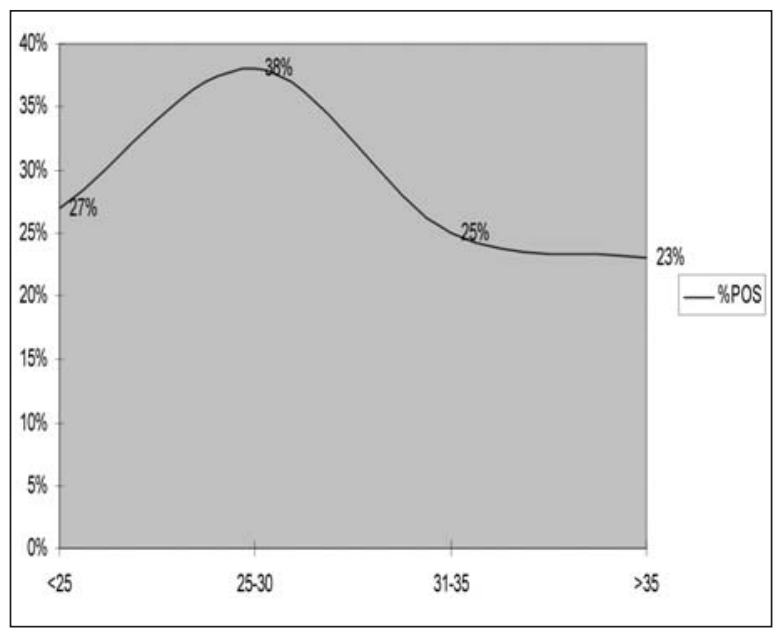

Figura I. Andamento della prevalenza dell'infezione divisa per fascia di età.

I dati mostrano che il $41 \%$ delle infezioni era sostenuta da un singolo ceppo, mentre nel 59\% dei casi si riscontavano infezioni miste causate da ceppi multipli.

L' $82 \%$ delle infezioni nella fascia di età $<25$ anni era sostenuta da ceppi multipli, mentre le altre fasce di età mostravano una prevalenza di infezione singole e miste in percentuale simile (Figura II).

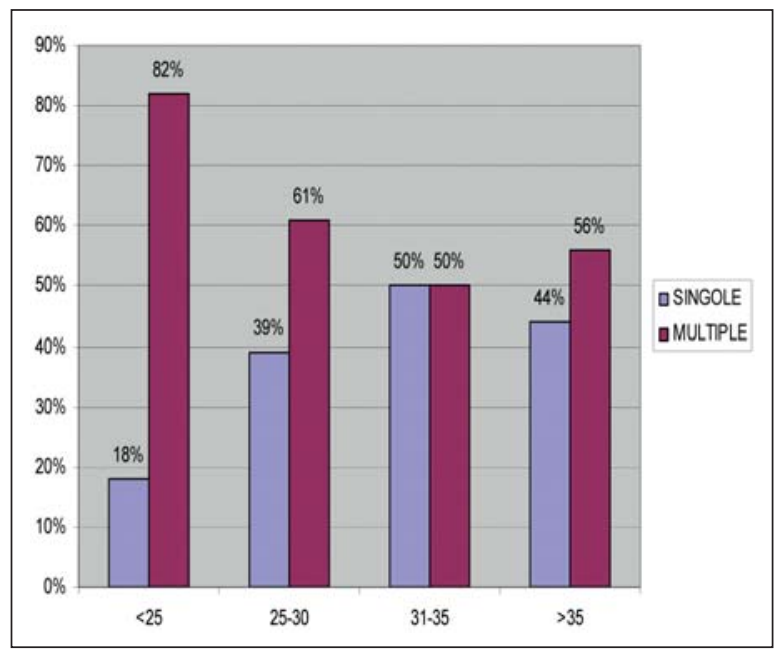

Figura II. Prevalenza di infezioni singole e miste.

L'analisi dei dati desunti dalle tipizzazioni genomiche HPV eseguite, ha evidenziato che i ceppi 33 (39\%), 31 (38\%), 58 (37\%) con maggiore frequenza sono stati identificati nelle infezioni singole o miste; mentre i ceppi ubiquitari 16 e 18 nella nostra realtà si presentavano rispettivamente nel $26 \%$ e nel $13 \%$ di tutte le infezioni diagnosticate (Figura III).

Nella nostra popolazione analizzata il microrganismo maggiormente isolato è stato Ureaplasma urealyticum (29\%) seguito dall'HPV (28\%) (Figura IV).L'isolamento in soggetti HPV-negati- 
vi non è infrequente: infatti, nel $45.5 \%$ dei campioni HPV-negativi veniva isolata almeno una specie microbica in contesto di dismicrobismo. Le specie maggiormente isolate sono state: Candida spp $(10.5 \%)$, S. agalactiae (13\%), Gardnerella vaginalis $(4.5 \%)$, E. coli (4\%) e Ureaplasma urealyticum (24\%) (Figura V). Generalmente quest'ultima specie è un frequente commensale del

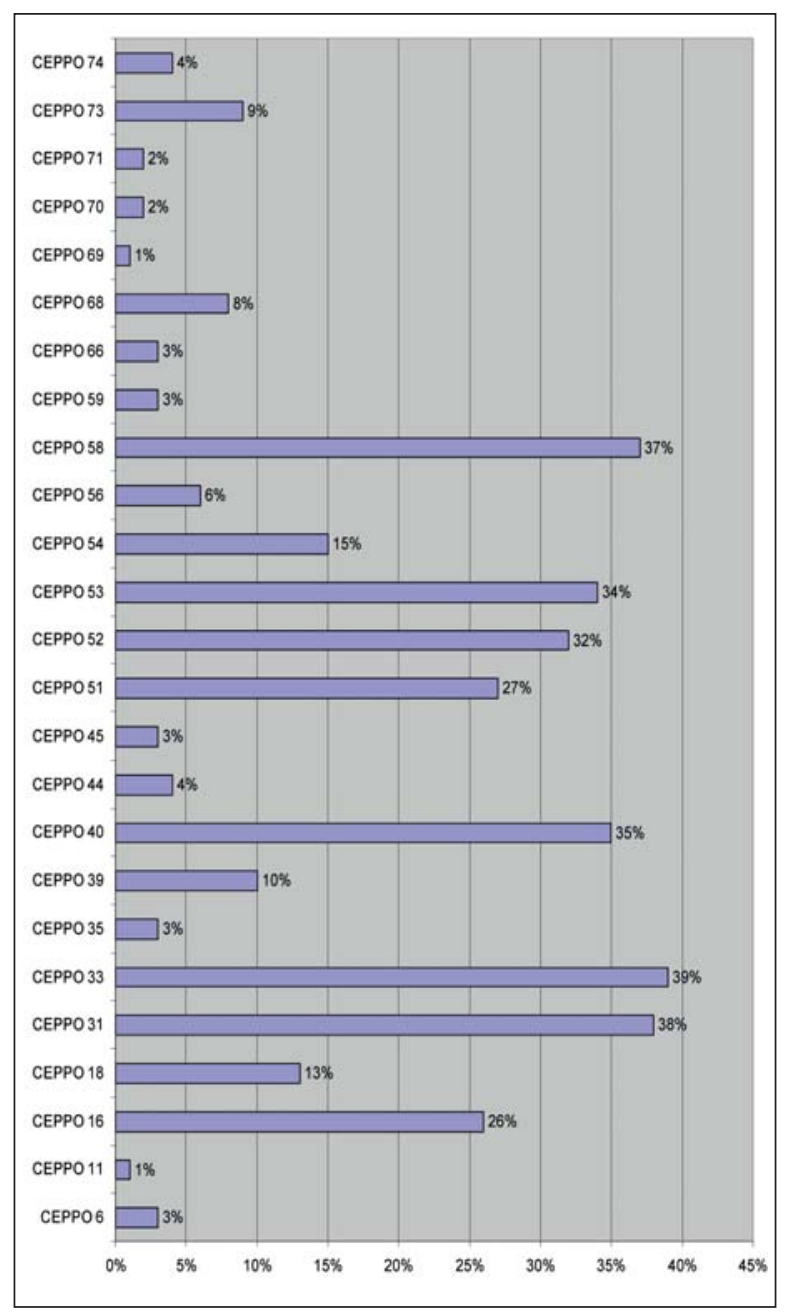

Figura III. Prevalenza dei diversi ceppi nelle infezioni singole e multiple.

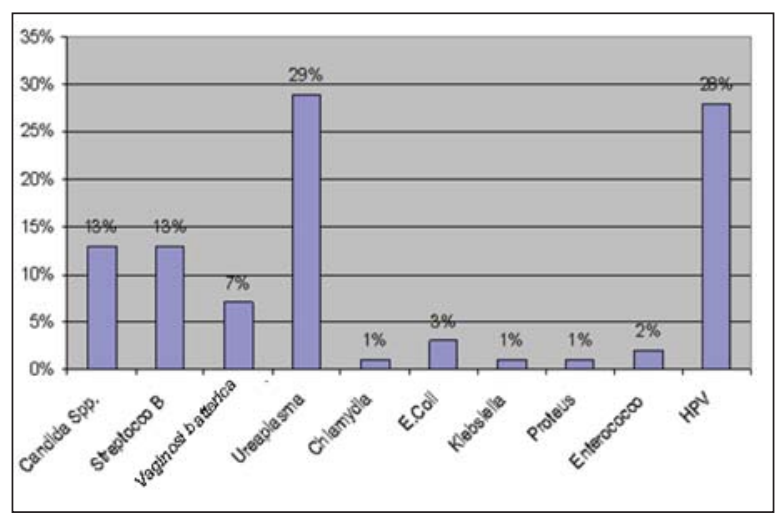

Figura IV. Prevalenza delle singole specie in contesto di dismicrobismo. basso tratto genitale femminile e sembra essere un importante patogeno opportunista durante la gravidanza e altre patologie genitali come le cerviciti (11).

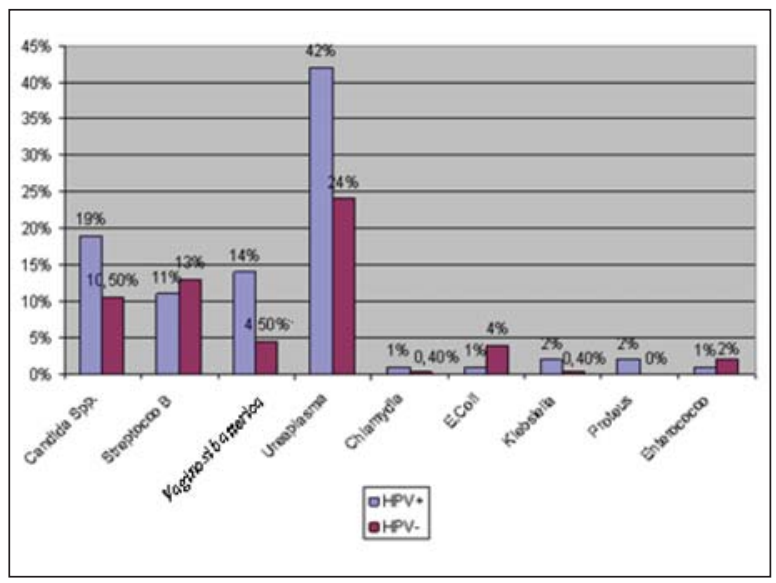

Figura V. Prevalenza di coinfezioni nelle donne HPV positive e negative.

Analizzando i dati di coinfezione, la presenza di almeno una specie microbica rilevata in pazienti HPV-positivi era evidente nel $66.7 \%$ dei casi. Nelle percentuali delle diverse specie microbiche rilevate in coinfezione con HPV si evidenzia ancora una volta il ruolo di primo piano svolto da Ureaplasma urealyticum (42\%) nell'ambito di tutte le coinfezioni.

L'odds ratio, come misura del rischio relativo è uguale a 2.4 .

In base al suddetto schema d'interpretazione, l'associazione coinfezione vaginale con la positività all'HPV $(\mathrm{OR}=2.4)$ è da classificarsi moderatamente probabile.

Al test del chi-quadro la differenza risulta significativa $(p<0.025)$.

Per quanto riguarda invece il rischio attribuibile, esso è pari a 0.17 . Ciò indica che rimuovendo il fattore "coinfezione vaginale" ci si aspetta di osservare una diminuzione del $17 \%$ dell'incidenza della malattia (ammesso che tale fattore sia l'unico responsabile dell'aumento d'infezione da HPV).

Al test del chi-quadro la differenza risulta significativa $(p<0.025)$.

Il rischio assoluto di contrarre l'HPV per un individuo esposto ad una coinfezione, che corrisponde all'incidenza dell'infezione da HPV nella popolazione esposta al fattore di rischio coinfezione, risulta essere del $36 \%$.

Analogamente il rischio assoluto di contrarre l'HPV per un individuo non esposto ad una coinfezione, coincide con l'incidenza dell'infezione da HPV nella popolazione non esposta al fattore di rischio coinfezione e nel nostro caso risulta 
essere del 19\%.

La coinfezione HPV/germi cervico vaginali è stata messa in relazione anche con il reperto citologico del PAP test. Infatti, allo scopo di valutare il ruolo svolto dalla coinfezione nella progressione displasica è stata valutata la prevalenza delle coinfezioni in relazione all'esito del PAP test (Figura V).

I dati mostrano come la coinfezione giochi un ruolo importante e progressivo procedendo con percentuali maggiori da ASCUS a L-SIL e H-SIL. Infatti, il $58.33 \%$, $1^{\prime} 85.71 \%$ e $88.89 \%$ rispettivamente dei casi di ASCUS, L-SIL e H-SIL presentavano una coinfezione HPV/U.urealyticum.

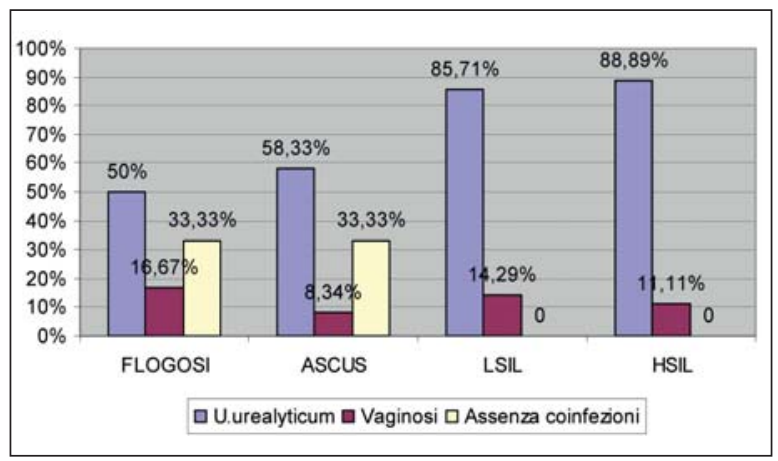

Figura VI. Coinfezione e progressione della displasia nella popolazione in studio.

\section{DISCUSSIONE}

Analizzando l'andamento delle positività per HPV (Figura I) si nota una maggiore prevalenza, pari al $38 \%$, nel range di età compreso tra 25 e 30 anni, età nella quale viene consigliato l'esecuzione dello screening del carcinoma della cervice mediante PAP test.

La percentuale di positività nella fascia al di sotto dei 25 anni è probabilmente sottostimata poiché l'esecuzione del PAP test non è consigliata e la diagnosi di una infezione spesso asintomatica passa inosservata a meno di complicanze dovute a coinfezioni da parte di microrganismi sessualmente trasmessi che impone l'esecuzione di un tampone cervicovaginale completo.

Inoltre la percentuale di positività è attenuata per effetto dei benefici derivanti dall'attuazione della campagna vaccinale che comincia a ridurre l'incidenza dell'infezione nelle giovani donne al di sotto dei 20 anni.

In accordo con i dati bibliografici (12) la percentuale di infezioni miste è maggiore nelle donne con età al di sotto di 25 anni in relazione $\mathrm{ad}$ una più intensa attività sessuale. Nella nostra popolazione la frequenza dei genotipi 31,33 , 40 e 58 è risultata maggiore rispetto ai ceppi ubiquitari 16 e 18 .
La coinfezione di almeno una specie microbica in contesto di dismicrobismo è stata rilevata nel $66.7 \%$ ed è risultata statisticamente significativa.

L'odds ratio, come misura del rischio relativo è uguale a $2.4(p<0.025)$ e da interpretare come moderatamente probabile.

Il rischio attribuibile $(\mathrm{RA}=0.17$ con $p<0.025)$ indica che rimuovendo il fattore "coinfezione vaginale" ci si aspetta di osservare una diminuzione del 17\% dell'incidenza dell'infezione da HPV (ammesso che tale fattore sia l'unico responsabile dell'aumento d'infezione da HPV).

Mentre il rischio assoluto risulta essere del 36\% negli esposti e del 19\% nei non esposti.

Studi epidemiologici hanno dimostrato il ruolo di altri agenti infettivi a trasmissione sessuale nella patogenesi del cancro della cervice, ma le conoscenze circa il ruolo specifico di questi patogeni nella storia naturale della infezione da HPV sono limitate $(5,8)$.

Sono stati descritti diversi meccanismi con cui queste coinfezioni possono agire, come la genotossicità diretta, ma probabilmente il meccanismo biologico più comune è l'induzione di un'infiammazione del collo dell'utero a causa di un aumentato stress ossidativo (4).

Nella popolazione oggetto di studio $U$. urealyticum potrebbe svolgere un ruolo importante nell'evoluzione delle infezioni HPV positive, essendo stato isolato in coinfezione nel $42 \%$ delle infezioni HPV positive.

$U$. urealyticum potrebbe essere un cofattore importante nello sviluppo delle displasie della cervice da HPV visto che la coinfezione aumenta in percentuale progressiva procedendo nel grado di displasia da ASCUS, L-SIL e H-SIL.

È stato segnalato che le infezioni da Mycoplasma causano in vitro delle variazioni cromosomiche e trasformazione delle cellule attraverso una perdita progressiva di unità geniche e induzione di traslocazioni cromosomiche (11).

Questi dati confermano che lo screening per le infezioni genitali può essere importante per rilevare la presenza simultanea di diversi microrganismi sessualmente trasmissibili.

Questi risultati suggeriscono e sottolineano l'importanza di eseguire lo screening per le infezioni cervico vaginali in pazienti HPV positivi al fine di eliminare $i$ probabili effetti sinergici derivanti dalle co-infezioni.

La prevenzione è importante non solo per evitare altre malattie sessualmente trasmissibili e le loro conseguenze, ma anche per ridurre l'influenza dei microrganismi sulla concomitante infezione da HPV. 


\section{BIBLIOGRAFIA}

1. Bornstein J, RMAAH. Etiology of cervical cancer: current concepts. Obstet Gynecol Surv. 1995; 50: 146-54.

2. Camargo-Campos AC, Freitas R, Ribeiro LFJ, Paulinelli RR, Reis C. Prevalence of vulvovaginitis and bacterial vaginosis in patients with koilocytosis. Sao Paulo Medical Journal. 2008; 126(6): 333-6.

3. Campos AC, Murta EF, Michelin MA, Reis C. Evaluation of Cytokines in Endocervical Secretion and Vaginal $\mathrm{pH}$ from Women with Bacterial Vaginosis or Human Papillomavirus. ISRN Obstet Gynecol. 2012; 2012: 342075. Epub 2012 Mar 22.

4. Castle PE, Giuliano AR. Genital travt infections, cervical inflammation, and antioxidant nutrient-assessing their roles as HPV cofactors. J Natl Cancer Inst Monogr 2003; 31: 29-34.

5. Clifford GM, Goncalves MA, Franceschi S. HPV and HIV Study Group. Human papillomavirus types among women infected with HIV: a meta-analysis. AIDS. 2006; 20(18): 2337-44.

6. Discacciati MG, Simões JA, Lopes ES, et al. Is bacterial vaginosis associated with squamous intraepithelial lesion of the uterine cervix? Diagnostic Cytopathology. 2006; 34(5): 323-5.

7. Dunne EF, Unger ER, Sternberg M, et al. Prevalence of HPV infection among females in the United States. Journal of the American Medical Association. 2007; 297(8): 813-9.

8. Flores R, Papenfuss M, Klimecki WT, Giuliano AR. Cross-sectional analysis of oncogenic HPV viral load and cervical intraepithelial neoplasia. Int $J$ Cancer. 2006; 118(5): 1187-93.

9. IARC Monographs on the evaluation of carcinogenic risks to humans, Vol. 90; Human Papillomaviruses. Lyon, France: International Agency for Research on Cancer; 2007.

10. King CC, Jamieson DJ, Wiener J. Bacterial vaginosis and the natural history of Human Papillomavirus. Infect Dis Obstet Gynecol. 2011.

11. Macpherson I, Russel W. Trasformation in hammer cells mediated by Mycoplasmas. Nature 1966; 210: 1342-5.

12. Mejhede N, Bonde J, Fomsgaard A. High frequency of multiple HPV types in cervical specimens from Danish women. APMIS 2009, 117: 108-14.
13. Murta EF, Souza MA, Araújo Júnior E, Adad SJ. Incidence of Gardnerella vaginalis, Candida spp and human papillomavirus in cytological smears. São Paulo Medical Journal. 2000; 118(4): 105-8.

14. Oliveira LHS, Ferreira MDPL, Augusto EF, et al. Human papillomavirus genotypes in asymptomatic young women from public schools in Rio de Janeiro, Brazil. Revista da Sociedade Brasileira de Medicina Tropical. 2010; 43(1): 4-8.

15. Platz-Christensen JJ, Mattsby-Baltzer I, Thomsen P, Wiqvist N. Endotoxin and interleukin- $1 \alpha$ in the cervical mucus and vaginal fluid of pregnant women with bacterial vaginosis. American Journal of Obstetrics and Gynecology. 1993; 169(5): 1161-6.

16. Scheurer ME, Tortolero-Luna G, Adler-Storthz K. Human papillomavirus infection: biology, epidemiology, and prevention. Int J Gynecol Cancer. 2005; 15: 727-46.

17. Schiffman MH, Castle P. Epidemiologic studies of a necessary causal risk factor: human papillomavirus infection and cervical neoplasia. J Natl Cancer Inst. 2003; 95: E2.

18. Silva CS, Adad SJ, Souza MAH, Barcelos ACM, Terra APS, Murta EFC. Increased frequency of bacterial vaginosis and Chlamydia trachomatis in pregnant women with human Papilloma virus infection. Gynecologic and Obstetric Investigation. 2004; 58: 189-93.

19. Sturm-Ramirez K, Gaye-Diallo A, Eisen G, Mboup S, Kanki PJ. High levels of tumor necrosis factor- $\alpha$ and interleukin- $1 \beta$ in bacterial vaginosis may increase susceptibility to human immunodeficiency virus. Journal of Infectious Diseases. 2000; 182(2): 467-73.

20. Vaccarella S, Herrero R, Dai M, et al. Reproductive factors, oral contraceptive use, and human papillomavirus infection: pooled analysis of the IARC HPV prevalence surveys. Cancer Epidemiol Biomarkers Prev. 2006; 15(11): 2148-53.

21. Verteramo R, Pierangeli A, Calzolari E, et al. Direct sequencing of HPV DNA detected in gynaecologic outpatients in Rome, Italy. Microbes Infect. 2006; 8(910): 2517-21.

22. Verteramo R, Pierangeli A, Mancini E, et al. Human Papillomaviruses and genital co-infections in gynaecological outpatients. BMC Infectious Diseases. 2009; 9 , article 16 . 